\title{
Consideraciones para el desarrollo de un sistema de evaluación de actividades académicas universitarias presentadas en formato audiovisual.
}

\author{
Esteban Manuel StePanian TARACIDo \\ Universidad Carlos III \\ estepani@hum.uc3m.es
}

Anto J. Benítez

Universidad Carlos III

abenitez@hum.uc3m.es

\begin{abstract}
Resumen:
De un sistema de evaluación se espera que resulte al menos eficaz, válido, coherente y objetivo. Condicionada o libremente, cada vez se presentan un mayor número actividades universitarias en formato audiovisual. Coincidiendo con un incremento de los enfoques prácticos, hace años que los procesos de evaluación en el Área de los estudios de Comunicación se asientan en la calificación de estos materiales, aunque no existe consenso en la forma de valorarlos. Los métodos más extendidos se han basado en matrices de evaluación o rúbricas, si bien se advierte una tendencia a incorporar en el proceso a los propios estudiantes a través de la coevaluación.
\end{abstract}

Palabras clave: EEES; enseñanza-aprendizaje; competencias; actividades en formato audiovisual; rúbrica; evaluación colaborativa

\section{Developing an assessment system for universitary activities in audiovisual} format

\begin{abstract}
:
An assessment system is expected to be effective, valid, consistent and objective. There are increasingly more university activities in audiovisual format. Coinciding with an increase in practices, for years types of assessment in the Area of Communication studies are based on the evaluation of these materials, although there is no consensus on how to value them. The most widespread methods have been based in rubrics, although there is a trend to involve students into the process through peer evaluation.
\end{abstract}

Key Words: EHEA; teaching-learning process; competences; audiovisual learning activities; rubric; peer evaluation.

\section{Referencia normalizada:}

Stepanian Taracido, E. M. y Benítez, A. J. (2014): Consideraciones para el desarrollo de un sistema de evaluación de actividades académicas universitarias presentadas en formato audiovisual. Historia y Comunicación Social. Vol. 19. Núm. Especial Febrero. Págs. 493-504.

Sumario: 1. Introducción. 1.1 Planteamiento. 1.2. El problema. 1.3. Objetivos. 2. Acerca de la metodología. 3. Características de la evaluación. 3.1. ¿Qué evaluar? 3.2. Método de evaluación. 3.3. La rúbrica como herramienta de evaluación. 4. La evaluación participativa. 5. Discusión y conclusiones. 6. Referencias bibliográficas. 


\section{Introducción}

El camino hacia el EEES ha incrementado, en el interior del proceso de enseñanza-aprendizaje, el uso de recursos docentes alternativos a los considerados como tradicionales en el pasado. Uno de ellos es el vídeo, ya asentado pero cuya utilización se intensifica en la actualidad con el fenómeno de los Massive Open Online Courses (MOOC).

Pese a esta presencia creciente, es menos habitual que los estudiantes realicen tareas académicas en vídeo. Y, a pesar de notables excepciones - por ejemplo, www. videolectures.net y www.jove.com-, tampoco abunda su empleo en la divulgación convencional de la investigación.

Al contrario, en el entorno de los estudios en Comunicación, debido a la orientación hacia la metodología práctica, cada vez es más común que se proponga a los alumnos un número de actividades a confeccionar y presentar en formato audiovisual.

Es previsible que el vídeo sea cada vez más utilizado, no solo como recurso de acceso al conocimiento y a la información, sino como fórmula de expresión de estudiantes y de investigadores en tareas académicas y divulgativas. Si esta previsión es cierta, se extenderá un problema que hace tiempo que afecta principalmente a los docentes en Comunicación Audiovisual, ya que no existe un procedimiento integral para evaluación este tipo de recursos.

\subsection{Planteamiento}

El nuevo paradigma de enseñanza-aprendizaje le da un papel central al estudiante. Este papel debe estar reforzado por un aumento en la conciencia de participación activa en los procesos de aprendizaje y de responsabilidad en su generación y aplicación (De Miguel, 2005: 42). De esta forma, una de las claves sería la valoración de los trabajos evaluables: "La evaluación sería una actividad imbricada en el propio aprendizaje. El profesor no será el único actor de la evaluación sino un copropietario cogestor de la misma”. (Ibid., 2005).

Aplicado este principio al ámbito de la Comunicación, la evaluación de las actividades que se han de presentar en formato audiovisual tendrá una gran influencia en la ruta formativa. Sin embargo, es una tarea complicada.

\subsection{El problema}

Un planteamiento práctico para producir vídeos necesita, para que sea viable, de un cierto grado de autonomía de los estudiantes, que suelen organizarse en pequeños grupos de rodaje durante los procesos de elaboración. En alguno de ellos, fuese la confección del guion o el rodaje de alguna secuencia en exteriores, el docente no podrá estar presente. Y no se trata tan solo de que exista una posibilidad de duda en la autoría real del trabajo, sino de medir la participación de cada uno de los estudiantes en las tareas efectuadas. Es preciso que los distintos equipos de rodaje en los que 
esté distribuido el grupo docente actúen simultáneamente y, por lo tanto, en un buen número de los procedimientos los únicos testigos serán los miembros del equipo. Estas no son circunstancias nuevas, ya que se han tratado, por ejemplo, en investigaciones que se ocupan de la docencia no presencial (Guardiola, 2011).

Es relevante también el problema de la conocida como 'alfabetización audiovisual', pero no solo en el sentido de saber leer e interpretar relatos construidos sobre soportes como el vídeo o el cine, sino también en el de saber expresarse de forma fluida y correcta con los recursos expresivos que poco a poco los artistas y artesanos audiovisuales han ido proponiendo y el espectador ha ido reconociendo como códigos comprensibles (Bonet, 2006; Ferrés, 2006). Si un problema real lo constituye el hecho de que los docentes se enfrentan a errores de ortografía y gramaticales en los trabajos escritos que proponen en mayor medida esto se repetirá en sus aspectos audiovisuales. Porque quizá sea más complicado establecer en qué medida es o no tolerable y afectará a la calificación de su trabajo el hecho de que se aparte de los estándares -muchas veces difusos, ciertamente- reconocidos como válidos en el entorno narrativo audiovisual.

En realidad, alrededor de la necesidad de estos estándares se halla gran parte del problema. No solo se trata de establecer lo que es válido o no, o lo que es cualitativamente discernible como positivo o como negativo, sino que el docente del área de Comunicación se enfrenta a la posibilidad de un trabajo que no funcione en lo concreto - por ejemplo, planos desenfocados, diferencias de luz en planos en la misma secuencia o errores espaciales en la narración- y que sea, debido a la impresión general que proporciona, aclamado por compañeros (al fin y al cabo, los 'pares' del vídeo juzgado) y testigos del visionado.

Si encontrar acuerdos comunes es complejo para reconocer y premiar indicios de calidad en el jurado de un certamen cinematográfico, ¿cómo esperar que dos docentes de la misma asignatura puedan otorgar la misma nota a un trabajo que se les presentase a ambos? ¿Es posible desarrollar un sistema eficaz, coherente, revisable y justo para trabajos académicos audiovisuales? Si no lo fuese, ¿cómo acercarse a un compromiso asumible?

Los procedimientos de evaluación tipo rúbrica aún no han demostrado, con certeza, capacidad para responder de un modo igual de eficaz al componente holístico de un vídeo, por lo que conlleva de elementos de juicio subjetivos, que a un número finito de criterios.

En estas condiciones, y pese a que la experiencia del docente pudiese sostener una evaluación sensata y argumentable, la sensación de injusticia o de improvisación sería difícil de eludir, por lo que la función de retorno que debería proporcionar la propia evaluación sobre la formación de los estudiantes quedaría puesta en duda.

En este sentido, los alumnos deberían ser capaces de prever los aspectos evaluables más débiles de su trabajo y de proyectar una calificación similar a la que cual- 
quiera de sus profesores le otorgaría: un autodiagnóstico que le permitiese analizar su evolución y mejorar su trabajo posterior.

\subsection{Objetivos}

Esta investigación se propone indagar acerca de la posibilidad de establecer un sistema de evaluación para actividades presentadas en formato audiovisual en el entorno universitario de la Comunicación. Este sistema debería, en un futuro próximo, conseguir conformar una serie de criterios y escalas asumibles como estándares objetivos; reducir la diferencia de percepción evaluativa entre los alumnos y los profesores a base de la participación directa de aquellos en los procesos de decisión de la génesis y la aplicación de la evaluación; valorar de forma coherente tanto los componentes concretos como los aspectos holísticos de las piezas en cuestión; e integrarse eficazmente en el sistema de enseñanza-aprendizaje, a fin de que los estudiantes se apoyasen en los resultados de la evaluación para seguir evolucionando positivamente.

\section{Acerca de la metodología}

Posiblemente por tratarse de un problema de naturaleza compleja y muy específico, una búsqueda bibliográfica que tenga como objeto encontrar documentos académicos que versen sobre evaluación de vídeos concebidos como actividades docentes universitarias dará como resultado un conjunto extremadamente escueto de textos.

Para realizar una revisión adecuada es preciso compartimentar el problema. Una consulta acerca de rúbricas aplicadas a las evaluaciones formativas revela el trabajo "Las rúbricas de evaluación como instrumento de aprendizaje entre pares", de Juan Pedro Navarro, Miguel Juan Ortells y Manuel Martí (2009), de la Universitat Jaume I de Castelló, que se acerca lo bastante al objeto de estudio como para ser considerado un antecedente necesario, si bien está enfocado a asignaturas de Humanidades o de Ciencias de la Educación.

La revisión, algo más general, de referencias acerca de la evaluación inter pares, de las aplicaciones formativas de los sistemas de evaluación -especialmente, de las rúbricas- y de la participación de los estudiantes en las actividades evaluativas -en el escenario de la centralidad del alumno y la organización docente a partir de las competencias- ha constituido un punto de apoyo para la reflexión sobre el caso que se propone, a pesar de su relativa rareza de uso: "discutir, compartir y consensuar a través de la coevaluación no es una práctica evaluativa que se realice en las aulas universitarias" (Rodríguez-Gómez et al., 2012: 15).

Además de la literatura académica, otras fuentes esenciales han sido las consultas de documentos, páginas y portales existentes sobre concepción y empleo de las rúbricas. Es posible encontrar en la red algunas muestras aplicables, como en el sitio 
Rubistar. Además, un ejemplo de rúbrica dedicada a narrativas digitales puede verse en Eduteka, en el que se ofrecen distinciones entre rúbricas aplicables a contenidos narrativos y a 'contenidos digitales'. Puede consultarse otro ejemplo, en uso también, publicado por la directora del Programa de Desarrollo Profesional Online de la Universidad de Wisconsin Joan Vandervelde (2010). Algunos de estos sitios web, incluso, invitan a variar criterios o baremos para construir una rúbrica propia y, también, a compartirla o a opinar sobre las ya publicadas.

También se ha desarrollado por profesores del Área de Comunicación Audiovisual de la Universidad Carlos III de Madrid una experiencia preliminar de evaluación, que no se considera suficientemente consistente como para incorporar sus resultados a la discusión, pero que corrobora los resultados respecto a la opinión de profesores y estudiantes sobre los beneficios de la autoevaluación de Valero-García et al. (2005) y de Rodríguez-Gómez et al. (2012). En dicha experiencia han participado los alumnos de un grupo de $3^{\circ}$ del Grado en Comunicación Audiovisual $(n=43)$, en equipos de 4 o 5 individuos. Se encargó a lo largo del cuatrimestre la elaboración de un portafolio a cada grupo, compuesto por tres piezas en vídeo de 1 a 2 minutos de duración. Los estudiantes debían atenerse a unos determinados condicionantes para desarrollar guiones, preparar las producciones, rodar y montar cada ejercicio.

A final del curso, antes de un pase en común de todas las piezas, se especificaba a los estudiantes los criterios y baremos de la rúbrica que debían utilizar para evaluar, y se les pedía que apuntasen lo necesario después de cada vídeo y que los evaluasen posteriormente en unos minutos al final del pase. Cada grupo debía, entonces, consensuar una nota para cada pieza -incluida una autoevaluación-, e indicar el conjunto de las notas individuales. La calificación emitida por el equipo para cada pieza no debía necesariamente ser una media ni mediana de las individuales, sino una consensuada, atendiendo también al nivel visto en general. De esta forma, se utilizaban al mismo tiempo la evaluación y la coevaluación como sistemas de calificación (Ahumada, 2006; Valero-García, 2005; Cavas et al. 2010), haciendo participar al estudiante en el sentido de Navarro et al. (2009: 2), es decir, con intención de poder obtener datos para diagnosticar y mejorar los procesos actuales.

$\mathrm{Al}$ final del pase, los estudiantes completaron un cuestionario con preguntas abiertas (Bravo et al., 2010) que contenía una sección acerca del sistema de evaluación del curso -adaptación de la propuesta de Navarro et al. (2009)-, en la que podían expresar qué opinión les merecía haber participado de esa manera en la evaluación.

Conviene indicar que el cuestionario se consideraba parte de la evaluación (es decir, en cierto modo, se hacía 'obligatorio') y que los estudiantes contestaban por medio de la plataforma virtual de la universidad, con lo cual la identidad del que contestaba era conocida. 


\section{Características de la evaluación}

La introducción del EEES y el progresivo deslizamiento del sistema universitario hacia una educación en competencias desde el paradigma clásico basado en la mera acumulación de conocimientos han complicado los sistemas de evaluación, que ya no se pueden limitar a comprobar si esos conocimientos han sido efectivamente memorizados por el alumno, sino que deben aspirar a verificar si han sido asimilados y están en disposición de ser aplicados a casos que emulen situaciones reales en contextos concretos.

Mientras que por su propia naturaleza la evaluación que tiende a comprobar la adquisición acumulativa de conocimientos es perfectamente compatible con un número muy reducido de pruebas o exámenes propuestos al finalizar el período docente, la evaluación de competencias adquiridas es preciso efectuarla de manera paralela a la propia ejecución de los ejercicios que el diseño curricular de la asignatura prevea a lo largo de todo el período docente.

El hecho de que el planteamiento y la ejecución de pruebas de competencias y su evaluación corran de forma paralela ha provocado que numerosos autores se hayan enfrentado al problema de planificar contenidos y objetivos de aprendizaje conjuntamente con sus sistemas de evaluación apropiados, para conseguir que estos últimos se puedan convertir en elementos integrados de la estrategia docente. (González Pérez, 2000; De Miguel, 2005; Ferrés, 2006.)

En realidad, se trata de evaluar el rendimiento del alumno en un verdadero proceso de enseñanza-aprendizaje continuo en el que la evaluación no se sitúe como una instancia de control externo sino como un factor esencial que pueda y deba contribuir al buen fin del proceso:

El nuevo paradigma focalizado en el aprendizaje del alumno implica necesariamente un papel activo y responsable por parte de éste y diluye la distinción entre lo que podemos considerar actividad de aprendizaje y actividad de evaluación. La evaluación sería una actividad imbricada en el propio aprendizaje. El profesor no será el único actor de la evaluación sino un copropietario cogestor de la misma. ${ }^{1}$

La ventaja fundamental de este planteamiento es que potencia los aspectos formativos de la evaluación frente a los puramente calificativos (Valero-García y Díaz de Cerio, 2005: 1) porque permite ofrecer a los alumnos informaciones precisas acerca de su proceso de aprendizaje: señalando sus problemas y carencias cuando está todavía a tiempo de resolverlas y destacando sus logros cuando el efecto favorable de la motivación puede ser eficaz. Es, además, un sistema muy útil para advertir al docente de los posibles defectos de su estrategia docente.

Sin embargo, asegurar estos beneficios exige, por un lado, una perfecta adecuación entre docencia y evaluación:

La eficacia de los procesos de enseñanza-aprendizaje está condicionada en buena medida por la eficacia de los sistemas de evaluación que se incorporen en ellos. Por otra parte, no puede haber unos sistemas de evaluación eficaces sin una definición 
precisa de los conocimientos, las habilidades y las actitudes que es preciso haber conseguido para poder considerarse competente en un ámbito académico ${ }^{2}$.

Y, por otro lado, exige también del docente una muy rápida respuesta evaluativa a cada una de las actividades del alumno. Para muchos autores la fiabilidad y la precisión son características esenciales de un buen sistema de evaluación, pero son precisamente estas dos características las que tienen un mayor coste de tiempo para el profesor que pretenda, como no puede ser de otra manera, administrarlas equitativamente. Sin embargo, la exigencia de prontitud en los planteamientos de evaluación continua es tan perentoria que para Valero-García y Díaz de Cerio (2005: 2) es razonable y asumible comprometer la precisión calificativa en aras de una agilidad evaluativa que permita asegurar la potencia formativa del sistema. "Lo importante es que el alumno sepa pronto si las decisiones importantes han sido acertadas. En cambio, no es excesivamente importante saber si el resultado merece una nota de 6,5 o una de 7" (Ibid.).

Porque el estudiante debe reaccionar con celeridad cuando (se) detecta que no alcanza las competencias exigidas.

Entre los inconvenientes cabe citar también el constante peligro de que el desarrollo de un sistema de evaluación rápido y eficaz que utilice las posibilidades de las plataformas informáticas de interacción con el alumno acabe por determinar los contenidos de la materia (Guardiola, 2011: 7).

Otro aspecto clave que se debe tener en cuenta en el planteamiento de una estrategia docente basada en un sistema de evaluación continua es el indudable carácter práctico que deben tener las pruebas que lo conformen. Desde luego, si consideramos las competencias como un conjunto integrado de "conocimientos, habilidades, actitudes y valores" (De Miguel, 2005: 105) debemos coincidir también en que

una competencia es algo que se demuestra en la acción, una potencialidad que se convierte en acto, que no es algo que se infiere sino algo que el estudiante hace y que está centrada en el desempeño profesional más que en lo científico-académico, debemos concluir que la planificación del proceso de enseñanza-aprendizaje debe asumir los principios de una metodología activa y práctica. ${ }^{3}$

Cuando se trata de pruebas realizadas en formato audiovisual es evidente que demostrar que se han adquirido las competencias exigidas pasa por la adquisición de un cierto grado de alfabetización audiovisual indispensable, aunque adecuada al área de conocimiento, como ya se ha visto más arriba. Reconocidos docentes de asignaturas ligadas a la creación audiovisual confirman la necesidad de que el alumno maneje con soltura los códigos de escritura audiovisual e insisten en que, dadas sus especiales características, solo ejercitándose en ellos se podrá lograr (Mackendrick, 2013: 33).

En concreto, en lo que se refiere a las asignaturas del área de Comunicación, existe el peligro de confundir la alfabetización en las técnicas de creación audiovisual con el aprendizaje de la operación de los equipos técnicos que la sustentan. Esta 
opción, considerada como fin último y desgraciadamente demasiado extendida, es absolutamente incompatible con el nivel exigible a los estudios universitarios. Ya se ha discutido la dificultad que tiene afrontar en ámbitos universitarios la formación mínima exigible que permita dominar las herramientas de producción audiovisual (Benítez y Stepanian, 2012). En todo caso, el adiestramiento en operaciones debe tener un carácter funcional e intermedio que permita desarrollar las capacidades intelectivas superiores a través del lenguaje audiovisual. ${ }^{4}$ Se trata de "dejar de «enseñar software»" y "alfabetizar digitalmente", en la feliz expresión de M. Bonet (2006: 8), porque "solo así se garantiza que los alumnos aprendan a aprender" (Ibid.).

\section{1 ¿Qué evaluar?}

Este texto no pretende abarcar la evaluación de todos los trabajos académicos en formato vídeo sino, tan solo, de aquellos que consideran los procesos creativos audiovisuales -léanse los procesos de conversión de la idea en guion, de producción, de puesta en escena y de puesta en pantalla- como su punto de interés para la calificación. Es preciso distinguirlos de los que utilizan los audiovisuales como medio de representación y que no solo no valoran el desempeño en las cuestiones de escritura en audio y vídeo, sino que advierten, de alguna manera, que la forma pudiese ocultar el fondo (Campos et al., 2010: 4). En términos radicales, remarcar esta distinción llevaría a recuperar un antiguo debate que no se quiere abordar en estas líneas -si la forma y el fondo son independientes o separables-, pero que resulta más o menos tolerable si los que así se expresan pertenecen a ámbitos externos a la Comunicación en los que existe más costumbre de presentar trabajos por escrito o los que van a aplicarlo son estudiantes de Medicina, como en el caso de la referencia anterior. Es pertinente recordar en este punto que la forma de medir la calidad de las propuestas audiovisuales consiste en observar su eficacia en los planos expresivo y comunicativo y no tan solo los elementos que tengan que ver con lo espectacular en su construcción.

\subsection{Método de evaluación}

En cuanto al método de evaluación, siempre en la misma área de Comunicación, es muy habitual que las pruebas se propongan a grupos de estudiantes (entre dos y cinco) a los que se les encomienda la elaboración de un portafolio que incluya el montaje final y la memoria de actividades de una producción audiovisual típica -sea ésta de ficción o de carácter informativo-.

Cada uno de los alumnos integrantes del grupo asume sus tareas propias en función de la figura profesional que le haya correspondido emular (realizador, productor, operador de sonido, director de fotografía...) para completar conjuntamente las fases del proceso productivo audiovisual.

Se trata de un "modelo centrado sobre la práctica" que, según De Miguel (2005: 157), se caracteriza porque tiene como objetivo final el desarrollo de competencias profesionales; se organiza en torno al estudio de casos prácticos y la resolución de problemas; y requiere procesos de evaluación acordes a su enfoque. 
De acuerdo con los intereses u objetivos docentes de la asignatura la valoración se centrará en unos u otros aspectos de los innumerables criterios que se podrían aplicar a un montaje audiovisual propuesto y realizado como una práctica docente. Eficacia comunicativa del guion; planteamiento adecuado de la luz; creación de un espacio sonoro coherente; planificación visual acorde; verosimilitud de la propuesta escenográfica; desempeño de los actores... son solo algunos de los muchos y muy variados ítems evaluables de una producción audiovisual.

Y, como se ha indicado antes, el problema es precisamente ese: evaluar y, en su caso, calificar adecuada y equitativamente las competencias exigidas y adquiridas por el alumno en la realización de una pieza audiovisual, seleccionándolas de entre los innumerables conceptos potencialmente valorables en otras asignaturas. Asegurando además la coherencia de resultados entre los posibles distintos docentes de los diversos grupos de la misma asignatura.

\subsection{La rúbrica como herramienta para evaluar}

Hasta el momento el instrumento preferido para asegurar la ecuanimidad de la evaluación parece ser la rúbrica. Se trata de una matriz organizada en la que se enfrentan los criterios evaluables con los baremos de calificación. La selección de los criterios, de acuerdo con los objetivos docentes de la materia, se realiza según un "sistema de categorías en los que se recogen claramente aquellos elementos susceptibles de ser evaluados y considerados como relevantes," (Navarro et al., 2009: 8). ${ }^{5}$

La aplicación de los baremos que califican desde el máximo nivel de desempeño hasta el más deficiente, se fundamentan en la consecución o no, por parte del alumno, de las competencias propuestas. Las rúbricas suelen devolver también comentarios justificativos, en muchos casos estandarizados, de la calificación obtenida en cada uno de los ítems evaluables.

\section{La evaluación participativa}

Son muchos los autores que destacan los aspectos positivos de implicar a los alumnos en la evaluación de sus propias propuestas. En particular Rodríguez-Gómez et al., aun reconociendo que no es práctica común en la enseñanza universitaria actual defienden "una nueva cultura de la evaluación en la que la actividad evaluativa llegue a ser por sí misma tarea de aprendizaje" (2012: 2) a través de la autoevaluación y la evaluación entre pares integradas en un sistema de coevaluación compartido con el docente.

Integrar al alumno en el sistema de enseñanza-aprendizaje como evaluador activo en colaboración con el profesor le permite mejorar su propio proceso de formación y su desempeño en competencias porque: 
mediante la educación en la evaluación se puede aprender a establecer criterios (y con ellos prioridades), a reflexionar sobre lo positivo y lo negativo de las realidades, a valorar (y comparar) los objetos de evaluación y sobre todo, a tomar decisiones fundamentadas y justificadas. De esta forma se puede favorecer que el estudiante autorregule su aprendizaje y fomentar su aprendizaje autónomo y, desde una perspectiva profesional, que se adapte más fácilmente a los cambios y esté capacitado para asumir responsabilidades. ${ }^{6}$

Un sistema de evaluación así planteado contribuye además a minimizar un problema -ya arriba apuntado- frecuente en los estudios de Comunicación Audiovisual. Es relativamente común que pruebas audiovisuales que reciben una evaluación modesta por parte del profesor alcancen un cierto éxito entre los propios alumnos del grupo cuando son puestas a disposición pública en plataformas on-line o redes sociales. La reducción de esta brecha, que produce una fuerte impresión de injusticia en el alumnado, se producirá tanto por el peso que la opinión de los alumnos tenga en la evaluación final, como por el hecho de que su responsabilidad como evaluadores les obligará a valorar de forma más precisa los objetivos docentes de la asignatura y ser más autocríticos con sus propias creaciones.

\section{Discusión y conclusiones}

Una evaluación adecuada de las actividades realizadas en formato audiovisual debería recurrir al concepto de "evaluación auténtica" en tanto que evaluación que toma como referentes los criterios de calidad del "mundo profesional" (De Miguel 2005: 157). Pero este principio se topa con el problema fundamental de que tampoco en contextos profesionales está ni mucho menos resuelta la cuestión de valorar con justicia la calidad de los productos audiovisuales. Si consideramos además que no todas las actividades evaluables deben provenir necesariamente del área de Comunicación deberíamos admitir que el sistema propuesto debería adaptarse a los diferentes grados de alfabetización audiovisual del alumno.

En todo caso la propuesta de un sistema de evaluación que responda a los objetivos arriba planteados debe tener carácter iterativo porque son los resultados de la evaluación los que deben permitir el correcto avance del alumno en su proceso de aprendizaje. Para ello debe asegurarse que los alumnos dispongan de estos resultados con la suficiente rapidez y que, además de las informaciones numéricas relativas a la calificación, se les suministren informaciones valorativas acerca de su desempeño.

Los instrumentos básicos de evaluación pueden ser rúbricas específicamente diseñadas con arreglo a los objetivos docentes de la materia en las que, dadas las especiales características de la expresión audiovisual, el componente holístico tenga suficiente presencia en los porcentajes de calificación. 
Se debe asegurar la presencia del alumno en el proceso a través de la aplicación de un sistema compuesto por tres instancias coevaluadoras: el propio alumno; sus pares y el profesor responsable.

\section{Bibliografía}

AHUMADA ACEVEDO, P. (2006). "Estrategias y procedimientos para una evaluación auténtica de los aprendizajes en la enseñanza universitaria”. En Cuadernos IRC 1-12. www.cbtis179.edu.mx/portal2/docentes/.../estrategias_evaluacion.pdf [14-08-2013]

BENÍTEZ, A. J., y STEPANIAN, E. M. (2012). "Desarrollo de las competencias específicas relacionadas con la tecnología para las áreas de periodismo y comunicación audiovisual”. En Estudios sobre el Mensaje Periodístico, n ${ }^{\circ}$ 18, p. 129-140.

BONET, M. (2006). "Centralidad de la comunicación audiovisual en el entorno digital : propuestas desde la experiencia formativa". En Revista de Universidad y Sociedad del Conocimiento, 3(2), p. 1-12.

BRAVO, E. et al. (2010). "An innovative teaching practice based on online channels: A qualitative approach". En World Journal on Educational Technology. $\mathrm{n}^{\mathrm{o}} 2$, p. 113-123.

CAVAS, M. et al. (2010): "La autoevaluación y la coevaluación como herramientas para la evaluación continua y la evaluación formativa en el marco del espacio europeo de Educación Superior". Comunicación presentada en las IV Jornadas de Innovación Educativa y Enseñanza Virtual en la Universidad de Málaga. $\mathrm{http} / / / \mathrm{www} . u m a . e s /$ ieducat/new_ieducat/IV_Jornadas_Comunicaciones/6_04.pdf [18-08-2013]

DE MIGUEL DÍAZ, M. (Dir.). (2005). Modalidades de enseñanza centradas en el desarrollo de competencias. Orientaciones para promover el cambio metodológico en el Espacio Europeo de Educación Superior. Universidad de Oviedo. Oviedo.

FERRÉS PRAT, J. (2006): "La competencia en comunicación audiovisual : propuesta articulada de dimensiones e indicadores". En Quaderns del CAC, 25, p. 9-17.

GONZÁLEZ PÉREZ, M. (2000). "Evaluación del aprendizaje en la enseñanza universitaria". En Revista Pedagogía Universitaria, 5(2), p. 31-61.

GUARDIOLA, J. (2011). "Algunas consideraciones sobre la evaluación de conocimientos jurídicos en plataformas virtuales". Ponencia presentada en las II Jornadas sobre docencia del Derecho y Tecnologías de la Información y la Comunicación. http://www.uoc.edu/symposia/dret_tic2011/pdf/4.guardiola_garcia_javier. pdf . [02-06-2013]

MACKENDRICK, A. (2013). On Film Making. Manual de escritura y realización cinematográfica. Ediciones Jaguar. Madrid.

NAVARRO, J. P.; ORTELLS, M. J. y MARTÍ, M (2009). Las rúbricas de evaluación como instrumento de aprendizaje entre pares. En GIL, M ${ }^{\mathrm{a}}$. D.; RAMÍREZ, M$^{\text {a }}$. I. y GIL, C. (eds.) (2009): Novena Jornada sobre Aprendizaje Cooperativo 
y Segunda Jornada sobre Innovación Docente. Universidad de Almería. Almería. p. 1-15. http://www.greidi.uva.es/JAC09-JID09/ficheros/ACTAS_JAC09.pdf [20-09-2013]

RODRÍGUEZ-GÓMEZ, G. et al. (2012). "La voz del estudiante en la evaluación del aprendizaje: un camino por recorrer en la universidad". En RELIEVE - Revista Electrónica de Investigación y Evaluación Educativa, 18(2), p. 1-21. http://ojs. uv.es/index.php/RELIEVE/article/view/1985 [20-06-2013].

VALERO-GARCİA, M., y DÍAZ de CERIO, L. M. (2005). "Autoevaluación y co-evaluación : estrategias para facilitar la evaluación continuada". Comunicación presentada en el Congreso Español de Docencia en Informática. UPC. Casteldefells.

VANDERVELDE, Joan (2010): Vídeo Project Rubric.

http://urbachc.org/ Macbeth_Project_Rubric_pdf.pdf. [10-07-2013].

http://www.eduteka.org/Rubistar.php3.[13-07-2013]

http://www.eduteka.org/NarracionesDigitales_Rubrica.php.[13-07-2013]

\section{Notas}

1 De Miguel, 2005: 44.

2 Ferrés, 2006: 10.

3 De Miguel, 2005: 105

4 Somos conscientes de que el uso de la expresión "lenguaje audiovisual" puede presuponer una toma de posición acrítica en un debate científico antiguo pero no resuelto. La utilizamos aquí únicamente para referirnos a las evidentes posibilidades de transmisión de significados y emociones que tiene el formato audiovisual.

5 Navarro et al. remiten a CONDE, A. y POZUELO, F. (2007): Las plantillas de evaluación (rúbrica) como instrumento para la evaluación. Un estudio de caso en el marco de la reforma de la enseñanza universitaria en el EEES. Investigación en la Escuela, 63, pp 77-90.

6 Rodríguez-Gómez et al. 2012: 2

\section{Los autores}

Esteban Manuel Stepanian Taracido es doctor en Ciencias de la Información por la Universidad Complutense de Madrid. Desarrolló gran parte de su vida profesional como realizador de televisión. Actualmente es profesor de "Teoría y Técnica de la Realización" y "Tecnologías y Técnicas del Audiovisual" en el Departamento de Periodismo y Comunicación Audiovisual de la Universidad Carlos III de Madrid. Es también profesor de "Realización Audiovisual" en la Facultad de Comunicación de la Universidad Antonio de Nebrija. Sus líneas de investigación principales se centran en el análisis de los valores expresivos de los elementos contituyentes del producto audiovisual. 
Anto J. Benítez es Licenciado y Doctor en Ciencias de la Información (1989 y 2006) por la Universidad Complutense de Madrid. Actualmente profesor en Teoría y Técnica de la Realización, Tecnologías y Técnicas del Audiovisual y Dirección de Cámara, en el Departamento de Periodismo y Comunicación Audiovisual de la Universidad Carlos III de Madrid. Profesor (1994-2001) en la Facultad de Publicidad del Colegio Universitario de Segovia, dependiente de la Universidad de Valladolid (antes de la Universidad Complutense). Realizador de televisión y productoras audiovisuales. 